\title{
Remote Pyramid-Shaped Phosphor Coating for Phosphor-Converted White LEDs
}

\author{
Sevda Hasan Abdullayeva,,2, Teymur Yashar Orujov ${ }^{1,2}$, Nahida Nazim Musayeva, ${ }^{1,2}$ \\ Rasim Baba Jabbarov1,2, Samir Kamal Orujov ${ }^{3}$ \\ ${ }^{1}$ Institute of Physics, Azerbaijan National Academy of Sciences, Baku, Azerbaijan \\ ${ }^{2}$ Research and Development Center for High Technologies, Ministry of Communications and High Technologies, Baku, \\ Azerbaijan \\ ${ }^{3}$ Sumgait State University, Sumgait, Azerbaijan \\ Email:nahida@mail.ru,timphysics1@gmail.com
}

How to cite this paper: Abdullayeva, S.H., Orujov, T.Y., Musayeva, N.N., Jabbarov, R.B. and Orujov, S.K. (2017) Remote Pyramid-Shaped Phosphor Coating for Phosphor-Converted White LEDs. World Journal of Nano Science and Engineering, 7, 17-24.

https://doi.org/10.4236/wjnse.2017.72002

Received: May 4, 2017

Accepted: June 18, 2017

Published: June 21, 2017

Copyright $\odot 2017$ by authors and Scientific Research Publishing Inc. This work is licensed under the Creative Commons Attribution International License (CC BY 4.0).

http://creativecommons.org/licenses/by/4.0/

\begin{abstract}
Increasing light extraction efficiency is an important task when it comes to manufacturing a powerful white light emitting diode with high luminous flux per watt. In this paper the fabrication of a pyramid-shaped 3-dimensional phosphor coating is reported. It is represented by a phosphor cover, shaped into an array of pyramid like formations. It is proposed that such a structure can improve the light extraction efficiency and the color distribution characteristics of any phosphor-converted white LED. The luminous flux and luminous efficacy are being studied as a function of the forward current across the die. It was found out that with this kind of technique it was possible to achieve an $8 \%-14 \%$ increase in the efficacy of the pc-LED. This increase of light output power is being attributed to the reduction of the phenomena of total internal reflection (TIR) inside the packaging module.
\end{abstract}

\section{Keywords}

Light Emitting Diode (LED), Chip-On Board (COB) Package,

Light Extraction Efficiency, Total Internal Reflection (TIR)

\section{Introduction}

In recent years, white light-emitting diodes have become essential lighting sources because of their high efficiency, reliability, and long lifetime compared to conventional lighting sources. Until around 2005 LEDs were mainly used as indicator light bulbs with the output power of $0.1 \mathrm{~mW}$. Only in the last decade LEDs started their journey to replace such light sources as incandescent and fluorescent lamps. To achieve this, companies such as Osram and Lumileds have worked on the improvement of their light output power and efficacy. Today 
LEDs give nearly $150 \mathrm{~lm} / \mathrm{W}$, and by 2020 the industry aims at $200 \mathrm{~lm} / \mathrm{W}$ for consumer lamps. Some aspects of LEDs still need to be improved to get closer to the goals set to leverage the capacity stored in the LED technology. To do so they have to refine the efficiency of the LED dies and thermal dissipation of produced heat.

White light emitting diodes combine blue emission from InGaN dies and the re-emissioned broad spectrum light from phosphors, where the phosphor is dispersed within the epoxy resin, surrounding the LED die. The $\mathrm{Y}_{3} \mathrm{Al}_{5} \mathrm{O}_{12}: \mathrm{Ce}^{3+}$ (YAG:Ce) comprises a significant portion of the phosphors used in LED white lighting market due to their broad emission spectrum, high absorption strength, excellent thermal quenching, high quantum efficiency, excellent chemical stability, and fast decay time. Speaking of an efficiency of an LED as a whole, it is comprised of three parts: the internal quantum efficiency of a die, phosphor conversion efficiency and package extraction efficiency.

The internal quantum efficiency (IQE) of the YAG:Ce micro-size powder phosphor (MPP) has reached over 90\% [1]. But there are two major drawbacks for current pc-LEDs in terms of package extraction efficiency. The first is light scattering caused by phosphor micro-sized particles. When the blue photons from the die strike the phosphor particles, some blue light will be scattered by the phosphor particles. Converted yellow emission photons are also scattered. A portion of scattered light flows in the backward direction towards the die; the backscattered light is not only unusable but also leads to reliability issues. The amount of this backward light varies and depends in part on the particle size of phosphors. The other drawback is that the yellow emission from the phosphor powder is isotropic. Although some backward light can be recovered by the reflector in current LED packages [2], there is still a portion of backward light that will be absorbed inside the package and further converted to heat. Heat generated in the package may cause the deterioration of encapsulant materials, affecting the performance of both the LED die and phosphor, leading to a decrease in the luminous efficacy over lifetime. Recent studies from research groups at Rensselaer Polytechnic Institute found that, under the in the process of obtain white light, about $40 \%$ of the light is transmitted outward of the phosphor layer and $61 \%$ of the light is reflected inward [3]. It is claimed that using scattered photon extraction (SPE) technique, luminous efficacy is increased by $60 \%$. In an effort to address the high scattering/reflection loss of micro-size phosphors there have been a number of methods proposed. A remote phosphor package [4], which employs the phosphor layer placed at a distanced from the die. This is supposed to lower the probability of light to be scattered back on the substrate surface. The scattered photon extraction package [5] employs a reflector between the die and the phosphor to reflect the light from the phosphor and recirculate it between the phosphor and the reflector cup. Ring remote phosphor structure [6] employs the phosphor layer placed around the die in a cylindrical shape. Also glass ceramic phosphors [7] and transparent polycrystalline ceramic plate phosphors [8], thin-film phosphors [9] and YAG:Ce nanophosphors in transparent matrix [10] all have been suggested recently to better utilize the emitted photons and neu- 
tralize the effect of heat on the encapsulating material.

Remote phosphors have shown positive results in minimizing light backscattering, but photons still tend to scatter backwards due to the flat surface of the phosphor layer. Therefore, it is necessary to develop a new morphologically modified YAG:Ce phosphor that can satisfy the requirements for high extraction pc-LEDs and be easily implemented in a pc-LED package. In this work, we are further improving the luminous flux and lumen efficacy of white pc-LEDs by shaping the pc-LED's phosphor layer into an array of pyramid-shaped cones, hollow on the inside. Accordingly, this will cause more blue light, coming from the blue die, tore-excite the phosphor due to the greater utilization of blue light, as it scatters inside the structure. Moreover, yellow light can be kept away from the dies, with a larger portion of it directed at the phosphor, thus preventing it from causing heating issues. All this should minimize the negative effect of light scattering on the LED dies and phosphor, thus increasing the final light output of the LED module.

\section{Experimental Process}

To manufacture the pyramid-shaped phosphor converting layer, and commercial YAG:Ce was used to mold the pyramid-shaped cones (Figure 1). Two silicon molds were shaped to obtain pyramid-like pattern and then epoxy was poured into those molds. YAG:Ce was mixed with the encapsulant in proportions of 1:10 and filled into those molds, which were pressed together to form a thin phosphor layer. The phosphor consisted of 25 pyramids ( 5 row and 5 columns), with the height of $4 \mathrm{~mm}$ and foot width of $4 \mathrm{~mm}$. It was cured in the furnace at $120^{\circ} \mathrm{C}$ to harden. Also, a conventional flat phosphor layer was prepared in order to compare the two phosphor coating techniques. The same phosphor composite was used to make both the $3 \mathrm{D}$ and conventional phosphor coatings, with the difference that the former was made flat instead of pyramid-cone shaped.

To mount the manufactured phosphor covers, a LED array consisting of 100 in series and 100 parallel dies was assembled. A chip on board (COB) MPCB was used to house100 GaIn Nbased blue dies with sizes of $1 \times 1 \mathrm{~mm}$ and emission wavelength of about $450 \mathrm{~nm}(\lambda=450 \mathrm{~nm})$, which were attached to the MPCB by conductive glue. The dies were electrically connected using an ultrasonic wirebonding machine by FK Delvotek (G5). Transparent silicon encapsulant was dispensed onto the PCB for the purpose of the die and wire protection.

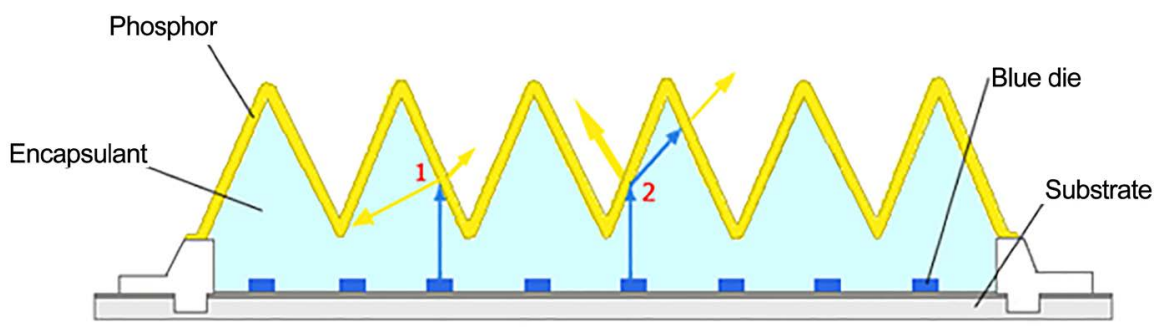

Figure 1. Explanation of the mechanisms of light interaction with the phoshor. 
Both 3D and flat phosphors were subsequently placed on top of the same LED array to compare their optical properties (Figure 2). In order to measure the electroluminescent (EL) properties, an integrating sphere by Everfine was used. A goniometer and a photo-colorimeter by Everfine were used to take the light distribution measurements.

\section{Results and Discussion}

In order to find out which of the pc-LED phosphor coating had a bigger light output, i.e. extraction efficiency, the current dependences of the luminous flux and luminous efficacy were measured. Figure 3 shows the current-dependent luminous efficacy and luminous flux of the 3D remote phosphor pc-LED and a conventional remote phosphor pc-LED. The luminous efficacy has decreased from $107 \mathrm{~lm} / \mathrm{W}$ to $68 \mathrm{~lm} / \mathrm{W}$ for the 3D phosphor LED and from $93 \mathrm{~lm} / \mathrm{W}$ to 65 $\mathrm{lm} / \mathrm{W}$ for the flat phosphor LED with an increase a applied current from 100 to

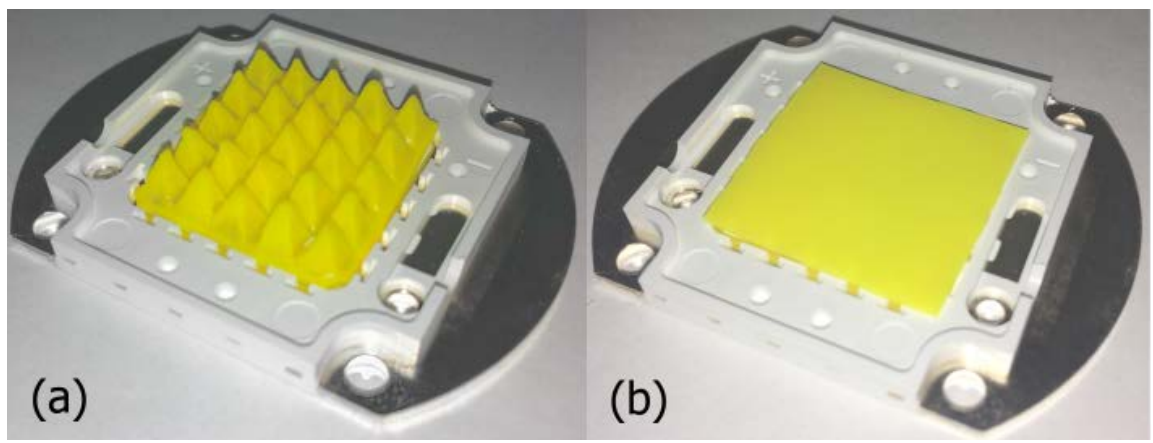

Figure 2. The overlook of the two phosphor covering techniques: (a) pyramid-shaped 3D phosphor; (b) conventional remote phosphor.

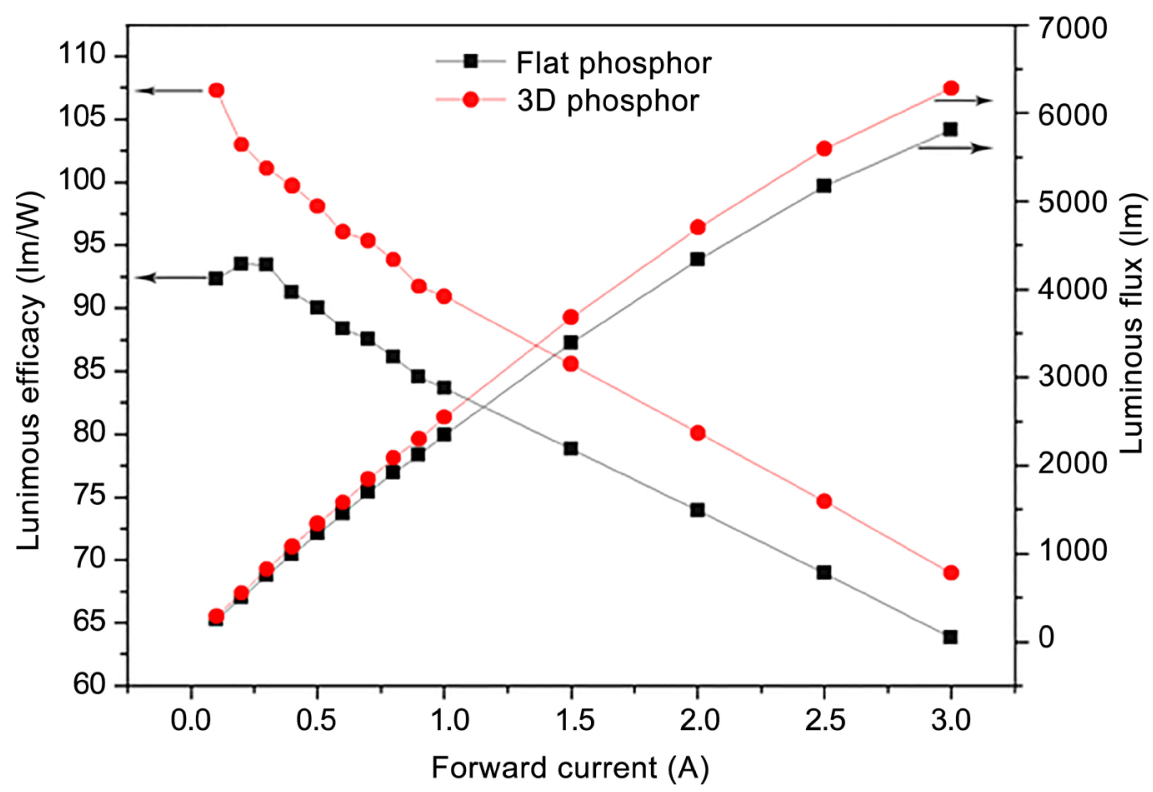

Figure 3. The current-dependent luminous efficacy and luminous flux of the pc-WLEDs with conventional remote phosphor and patterned remote phosphor structures. 
$3000 \mathrm{~mA}$. This decrease can be attributed to the efficiency droop of YAG:Ce phosphor, caused by the heat from the die, as well as by an increased number of non-radiative recombinations in the dies.

However, the 3D phosphor pc-LED showed an incremental increase in efficacy at all currents, and as the forward current increased the efficiency increment also rose. An $8 \%$ increase was observed at $100 \mathrm{~mA}$ and $14 \%$ increase at $3000 \mathrm{~mA}$. The enhancement of luminous flux was close to $8 \%$ at lower currents and close to $10 \%$ at its maximum, with these two LEDs' correlated color temperatures being $4324 \mathrm{~K}$ for 3D and $4644 \mathrm{~K}$ for the conventional LED. It should be noted that thicker phosphor yields higher luminous flux with all other parameters being equal. The samples were measured multiple times in order to determine the error probability, and it was found out that error comprises $0.5 \%$ of the measured values.

The reason for this boost in luminous efficiency was the enhancement of utilization of the blue light, so that an increased number of blue photons were absorbed and down-converted by the specifically shaped phosphor structure. At the same time, there was a higher probability of photons escaping from a refractive index gradient of the phosphor layer. In the conventional encapsulation layer packaging, due to the total internal retraction effect (TIR), a large mass of light is reflected at the interface between air and the encapsulation layer, and propagates toward the packaging substrate [11]. The pyramid structure deals with these problems. Looking at Figure 1, there are two types of interaction of light with the phosphor "1" and "2". "1" shows the absorption of blue photon and emission of a yellow photon. A portion of emitted yellow light is emitted into the visible light which is mixed with blue light, another portion is emitted backwards. In case of the $3 \mathrm{D}$ pyramid structure, because of its shape, a large margin of light is reversed, canceling the interaction with the dies. In case "2" blue photon scatters against the phosphor surface and as a result of an elastic collision cannot be converted into yellow light, returning into the package. The relation between the angles of the scattered photon is given by Snell's law:

$$
n_{1} \sin \sin \theta_{i}=n_{2} \sin \sin \theta_{t}
$$

With this angle, the scattered blue photons get redirected, and can potentially be reabsorbed by the phosphor layer. If we compare our results to similar works, in [12] they were able to achieve a $10.5 \%$ increase of light output power by implementing double layers with different interface shapes in between. While [13] reports that by using a polymer directly lens on the die, and quantum dot phosphor polymer layer distanced from it, a $29.7 \%$ increase in efficacy was achieved. And in [14] authors were able to increase the pc-LED's efficacy by $3.27 \%$ by forming nanosized moth-eye patterns on the surface of an LED package.

The angular dependence of the LEDs' CCT has also been investigated, as large variations of the CCT with viewing angle are not desirable in LED lighting applications. Figure 4 shows the angular dependence of CCTs of two different LEDs at the currents of $350 \mathrm{~mA}$. Now due to the fact that the phosphor layer in case of the 3D phosphor basically lies at an angle in relation to the dies, it might 


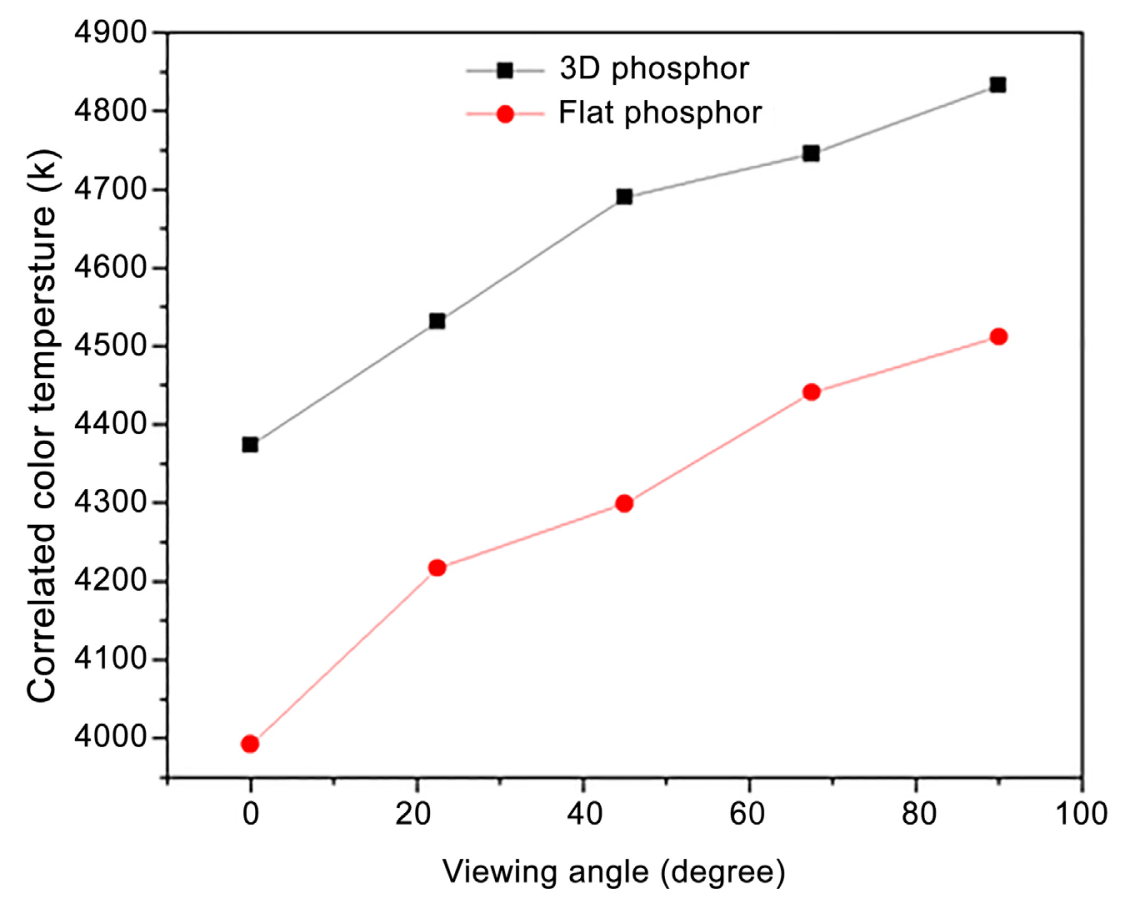

Figure 4. CCT as a function of the viewing angle for a conventional YAG:Ce CPP-capped LED and a 2D.
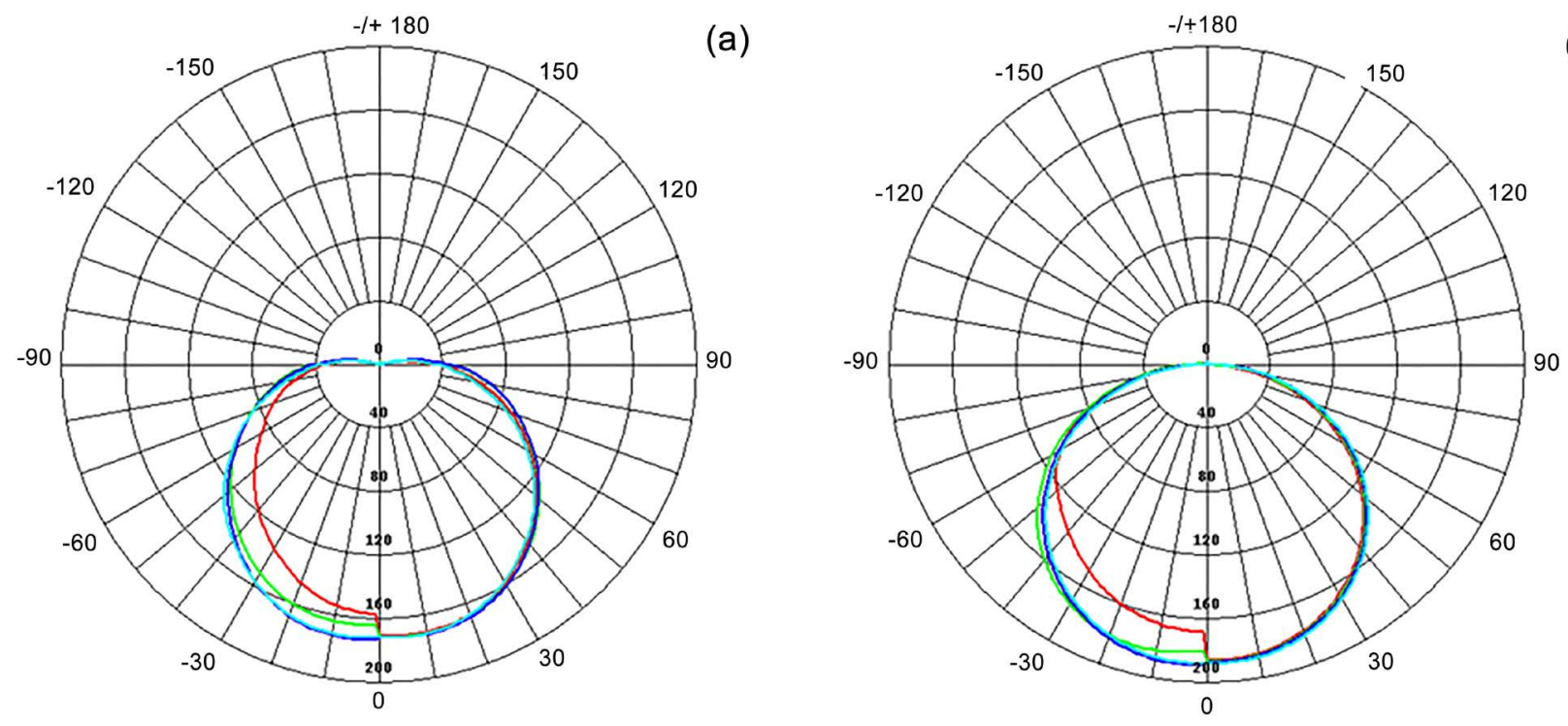

(b)

Figure 5. Light distributionof the conventional and manufactured 3D phosphor converted LEDs: (a) conventional phosphor pc-LED; (b) pyramid structured phosphor pc-LED.

be quite expected that CCT would improve when viewed from the side. In the conventional phosphor layer when the blue photons propagate too far into the depth of the phosphor, which leads to large angle diffraction of photons, the portion of down-converted yellow light will rise due to longer light path and higher absorption percentage of phosphor. If the yellow photons increase in number, a lower CCT light will be observed around the edges of the package, 
which is called the "yellow ring". But in case of the pyramid-shaped phosphor that portion of light propagating side wards, is not absorbed much deep in the phosphor bulk, because there is no horizontal thickness. And as a result the converted yellow rays are isotropically emitted from the phosphor particles. While experimenting with the thickness of the phosphor layer on the pyramids, it was discovered that the thicker the phosphor layer is the more stable the color stays across the perimeter of the package. The graph shows the two phosphor coating techniques having quite close variation of CCT depending on the viewing angle. In Figure 2, if we consider the angular-dependent CCT from 0 to 90 degrees, the CCT deviations of the conventional remote phosphor and 3D remote phosphor structures were $520 \mathrm{~K}$ and $459 \mathrm{~K}$, respectively. Figure 5 shows the light distribution of the measured relative lumens as a function of the viewing angle for two different LEDs, where red line is attributed to-C0/C180, greenC30/C210, dark blue-C60/C240, light blue-C90/C270 planes. Measurements were taken under identical forward currents of $350 \mathrm{~mA}$. Both conventional phosphor and 3D phosphor LEDs show similar distribution patterns in all dimensions. In both cases the light distribution is symmetrical around 90 degrees. The normally directed emission is about $87 \%$ higher for the $3 \mathrm{D}$ phosphor LED than the conventional remote phosphor LED. This means that the emission directed forward will be more intense, whereas the side emission will basically remain the same.

\section{Conclusion}

In this study, the pyramid shaped 3D remote phosphor covering technique was proposed and compared to a conventional flat remote phosphor coating. It was shown that by shaping the phosphor in a triangular pyramid-shaped way, it was possible to achieve a better light extraction from the package of pc-LED's surface of the phosphor. An 8\% - 14\% enhancement of luminous efficacy was observed as compared to the traditional pc-LED's phosphor covering. This efficacy growth was attributed to the reuse of backscattered photons, leading to more photons being absorbed by the phosphor particles and converted to emitted light. Moreover, a bigger phosphor emitting area could have also contributed to the increased luminous flux. The 3D pc-LED also showed good angle dependent correlated color temperature characteristics, which were better than that of the conventional pc-LED, as well as the good luminous intensity distribution. With the implementation of this novel design of phosphor converting layers, together with the use of other extraction enhancing techniques, we believe that it will be possible to reduce the losses in pc-LED packages to minimum values.

\section{References}

[1] Gorrotxategi, P., Consonni, M. and Gasse, A. (2015) Optical Efficiency Characterization of LED Phosphors Using a Double Integrating Sphere System. Journal of Solid State Lighting, 2, 1-14. https://doi.org/10.1186/s40539-014-0020-7

[2] Steigerwald, D.A., Bhat, J.C., Collins, D., Fletcher, R.M., Holcomb, M.O., Ludowise, 
M.J., Martin, P.S. and Rudaz, S.L. (2002) Illumination with Solid State Lighting Technology. Journal of Selected Topics Quantum Electron, 8, 310-320. https://doi.org/10.1109/2944.999186

[3] Zheng, Y. and Stough, M. (2008) White LED with High Package Extraction Efficiency. Osram Sylvania Product Inc. Central Research and Service Laboratory, Wilmington. https://doi.org/10.2172/963890

[4] Narendran, N. (2005) Improved Performance White LED. Fifth International Conference on Solid State Lighting, San Diego, 31 July 2000, 45-50. https://doi.org/10.1117/12.625921

[5] Narendran, N., Gu, Y., Freyssinier-Nova, J.P. and Zhu, Y. (2005) Extracting Phosphor-Scattered Photons to Improve White LED EFFIciency. Physica Status Solidi(a), 202, 60-62. https://doi.org/10.1002/pssa.200510015

[6] Lin, M., Ying, S.P., Lin, M.Y., Tai, K.Y., Tai, S.C., Liu, C.H., Chen, J.C. and Sun, C.C. (2010) Ring Remote Phosphor Structure for Phosphor-Converted White LEDs. IEEE Photonics Technology Letters, 22, 574-576. https://doi.org/10.1109/LPT.2010.2043088

[7] Chen, D., Xiang, W., Liang, X., Zhong, J., Yu, H., Ding, M., Lu, H. and Ji, Z. (2015) Advances in Transparent Glass-Ceramic Phosphors for White Light-Emitting Diodes-A Review. Journal of the European Ceramic Society, 35, 859-869. https://doi.org/10.1016/j.jeurceramsoc.2014.10.002

[8] Nishiura, S., Tanabe, S., Fujioka, K. and Fujimoto, Y. (2011) Properties of Transparent Ce:YAG Ceramic Phosphors for White LED. Optical Materials, 33, 688-691. https://doi.org/10.1016/j.optmat.2010.06.005

[9] Chao, W.-H., Wu, R.-J. and Wu, T.-B. (2010) Structural and Luminescent Properties of YAG:Ce Thin Film Phosphor. Journal of Alloys and Compounds, 506, 98-102. https://doi.org/10.1016/j.jallcom.2010.04.136

[10] Nyman, M., Shea-Rohwer, L.E., Martin, J.E. and Provencio, P. (2009) Nano-YAG:Ce Mechanisms of Growth and Epoxy-Encapsulation. Chemistry of Materials, 21, 1536-1542. https://doi.org/10.1021/cm803137h

[11] Sun, C.C., Chang, Y., Yang, T.H., Chung, T.Y., Chen, C.C., Lee, T., Li, D., Lu, C., Ting, Z.Y., Glorieux, B., Chen, Y.C., Lai, K.Y. and Liu, C.Y. (2014) Packaging Efficiency in Phosphor-Converted White LEDs and Its Impact to the Limit of Luminous Efficacy. Journal of Solid State Lighting, 1, 1-17.

https://doi.org/10.1186/s40539-014-0019-0

[12] Choi, H.S. and Moon, C.H. (2016) Improvement of the Light Extraction Efficiency of LED Package Using Double-Layered Encapsulant with Curved Interface. Journal of Nanoelectronics and Optoelectronics, 11, 170-174(5).

[13] Shin, M.H., Hong, H.G., Kim, H.J. and Kim, Y.J. (2014) Enhancement of Optical Extraction Efficiency in White LED Package with Quantum Dot Phosphors and Air-Gap Structure. Applied Physics Express, 7, No. 052101. https://doi.org/10.7567/apex.7.052101

[14] Shin, J.H., Choi, H.J., Han, K.S., Ra, S., Choi, K.W. and Lee, H. (2013) Effect of Anti-Reflective Nano-Patterns on LED Package. Current Applied Physics, 13, 93-97. https://doi.org/10.1016/j.cap.2013.01.014 
Submit or recommend next manuscript to SCIRP and we will provide best service for you:

Accepting pre-submission inquiries through Email, Facebook, LinkedIn, Twitter, etc. A wide selection of journals (inclusive of 9 subjects, more than 200 journals)

Providing 24-hour high-quality service

User-friendly online submission system

Fair and swift peer-review system

Efficient typesetting and proofreading procedure

Display of the result of downloads and visits, as well as the number of cited articles Maximum dissemination of your research work

Submit your manuscript at: http://papersubmission.scirp.org/

Or contact winse@scirp.org 